\title{
covid-19 Teleclinics in rheumatology introduced during the first lockdown phase of the COVID-19 pandemic of 2020
}

\author{
Authors: Antoni Chan, ${ }^{A}$ Annabel Suarez, ${ }^{B}$ Joanne Kitchen ${ }^{C}$ and Anthony Bradlow ${ }^{C}$
}

The COVID-19 pandemic has led to a crisis in the provision of usual services, including face-to-face (FTF) outpatient clinics. The need for change came abruptly in late March 2020 as routine clinics were cancelled. We accelerated the delivery of our teleclinics (telephone and video) in rheumatology, which we had started doing prior to the pandemic. 396 patients were reviewed during the lockdown by teleclinics. 39 (10\%) of patients were discharged, $102(26 \%)$ had treatment adjusted without bringing forward their appointment, 39 (10\%) had tests ordered and prescriptions issued, 160 (40\%) were reviewed and booked for a future appointment, thus reducing the current waiting list, and $22(6 \%)$ were placed a patient-initiated followup plan. Only $10(3 \%)$ required a conversion from the teleclinic to a FTF clinic in the near future. The COVID-19 crisis offered us the opportunity to do things we had been considering for some time. Our aim is now to continue with this new way of working as we move to the recovery phase and beyond. We would suggest that adoption of these changes in other trusts could significantly improve the quality of the care for patients not only in rheumatology but also throughout the NHS.

KEYWORDS: teleclinics, virtual clinics, service improvement, digital

DOI: 10.7861/fhj.2020-0093

\section{Introduction}

The COVID-19 pandemic resulted in disruption of the usual delivery of clinical services, including face-to-face (FTF) clinics and elective procedures. Redeployment, sickness and shielding reduced staff availability. Patients were advised to stay at home, thus making FTF contact with practitioners challenging.

We needed to keep the rheumatology service going to ensure that patients with long-term rheumatic conditions were treated and monitored during the pandemic. Patients also continued to phone the department seeking advice on their condition, so a

Authors: Aconsultant rheumatologist and associate medical director, Royal Berkshire Hospital, Reading, UK; B specialist registrar, Royal Berkshire Hospital, Reading, UK; ' Consultant rheumatologist, Royal Berkshire Hospital, Reading, UK decisive response was needed. We thus accelerated the delivery of teleclinics (telephone and video) which we had initiated on a small scale prior to the pandemic.

\section{Aims}

The aims of this paper are to report on our experience of delivering teleclinics during the COVID-19 pandemic and on our plans to sustain this delivery long term. We outline the benefits and risks of teleclinics, inclusion and exclusion criteria, prerequisites for successful teleclinics, and guidance on how to conduct and follow up teleclinics.

\section{Methods}

Rapid delivery and roll out of teleclinics

With the COVID-19 crisis in March 2020, an early decision was made by our Trust to start teleclinics. Staff who were self-isolating considered ways of taking the load from those working in the frontline. For those in the frontline, teleclinics allowed flexibility in conducting clinics in the face of a fluid and fast-changing ward environment.

The teleclinic set up underwent a privacy impact assessment (PIA) in line with the General Data Protection Regulation (GDPR) Act. The process of data collection and access to patient/ physician identity were approved by the Trust Information Governance Committee prior to the delivery of the teleclinics. Good information technology (IT) support ensuring good network connection and the availability of clinical documentation, pathology and radiology results all in one place with a single login was key in ensuring successful rapid delivery of teleclinics. Steps were taken to increase capacity for remote login to the electronic patient record (EPR) and other programmes for offsite working. New SIM cards were issued for phone conversations with patients and others. The video software NHS Attend Anywhere was quickly deployed across the Trust. Group conferencing software (Windows Teams) was downloaded onto Trust laptops used at home.

We provided in-house training to all staff at the start of the pandemic. 'How to' guides were produced and access to training materials for the software was given.

All the virtual clinics were recorded and run through the EPR, as were the documentation and ordering of investigations, with results were returned to the clinician's result folder through the 
Box 1. The practicalities of setting up teleclinics

> Agree on the criteria for patients suitable for teleclinics (see inclusion and exclusion criteria in Box 2)

> Prospectively triage all existing follow-up clinic lists online and stream patients into teleclinics based on the agreed criteria

> The administrative team should set up the teleclinic on the electronic patient record (EPR) for record of clinical activity and for coding. Before the clinic, they should notify patients of the date of teleclinics by email, text or phone call, checking the phone number in the EPR against the NHS Spine, which is more accurate. It is essential to be able to contact each patient easily and accurately on the day. Video consultations need to be agreed with the patient in advance, usually by prior telephone call, in case they do not have email and access to the internet. This will become even more important after the easing of restrictions linked to the COVID-19 pandemic when fewer people are confined to home

$>$ Each practitioner will need

$>$ a good home internet connection and a trust SIM card if calling from home

> a headphone with microphone or speakerphone to allow typing into the EPR during the consultation

$>$ two computer screens for videoconsulting - one to contact and see the patient and the other linked to the EPR and PACS system for $\mathrm{X}$-ray reviews

Virtual Clinic episode on EPR. Blood test forms and prescriptions were sent to patients directly at home with their clinic letter from the virtual clinic. Patients could attend their GP surgery to have blood tests and collect or have their prescription delivered to them at home.

Our departmental administrative team has provided key system support, such as confirming patient details and setting up appointments. Some administrative teams are also proactively scanning future clinics to offer patients the option of teleclinics. This has been recorded on the EPR which improves accuracy in coding and activity data.

Patients were notified of their teleclinic appointment by email, text or phone and requested to have an up-to-date list of their medications ready for the teleclinic. The practicalities of setting up teleclinics rapidly and urgently in during the COVID-19 pandemic in March 2020 are shown in Box 1.

Triage and streaming of both new referrals and existing follow up lists are important to ensure that the right patients are seen in the right setting. The administrative team have accessed all referral letters and follow up clinic lists. Practitioners not in the frontline were tasked to triage and stream patients to teleclinics. For this purpose, a list of inclusion and exclusion criteria for patients suitable for teleclinics was developed. This is shown in Box 2.

Structured preparation by the practitioner before the teleclinic is essential in order to maximise the consultation time during the teleclinic. A suggested preparation structure is shown in Box 3 .

\section{Results}

During the period 18 March to 25 May 2020 (10 weeks), all four authors ran teleclinics in rheumatology. Two authors were also involved with inpatient COVID-19 ward duties.

During this period, $\mathrm{n}=396$ patients were reviewed through teleclinics. Of these, 309 (78\%) were telephone and 87 (22\%)
Box 2. Inclusion and exclusion criteria for teleclinics

\section{Inclusion criteria}

> Patients whose condition is clinically stable with low disease activity scores, who are making good progress and doing well on disease modifying drugs (DMARDs) or biologics

> Patients who already have a wide appointment interval, eg 12 months, and in whom not much new has happened between appointments

> Patients requiring discussion of test results and proposed treatments/drugs after initial appointment

> Osteoporosis referrals where patients require interpretation of DEXA and advice about treatment

> Alternate clinics for patients requiring monthly escalation for early inflammatory arthritis

> Patients requesting to be seen earlier than their set appointment this allows accurate assessment of the degree of urgency required

> Patients on remote monitoring who are completing their outcome scores online and with low disease activity

> Patients not suitable for patient-initiated follow up, where a teleclinic will enable assessment of their condition

\section{Exclusion criteria}

$>$ Patients who decline to have teleconsultation

$>$ Patients not in a location where they can speak confidentially

$>$ New patients being referred with a new problem; they should have face-to-face (FTF) appointments unless there is a good reason for a teleclinic (eg symptoms suggest that accurate advice can be given in a teleclinic)

> Patients with new symptoms that need clinical examination for accurate evaluation

$>$ Patients with existing conditions that need clinical examination for meaningful assessment, eg swollen joint counts in rheumatoid arthritis

> Situations where patient confidence requires FTF consultation even if appropriate decisions could be made in a teleclinic. Often such patients require the reassurance of a clinical examination

$>$ Children under the age of 18 , unless a parent or guardian is available, and vulnerable adults

> Patients who are unable to use or access IT or phone

$>$ Patients with communication difficulties, eg speech/hearing impairments, poor English if independent interpreter service not accessible

$>$ Patients with impaired cognition, unless a relative or friend is available to speak on patient's behalf with patient's adequate consent

were video clinics. The number of patients seen represented just under $50 \%$ of the total workload during the timeframe of the study. For the first half of the study there were no FTF appointments. FTF appointments were gradually introduced in the second half of the study.

The mean age (range) was 58.9 years (17-92). The majority of patients were attending follow-ups $(n=350,88 \%)$ and the remainder were new referrals $(n=40,12 \%$ ). Patient demographics, disease categories and teleclinic outcomes are summarised in Table 1.

\section{Discussion}

In recent years, as technology has improved and as pressures have grown on traditional outpatient consulting systems, interest in teleconsulting (both video and telephone consultations) has 
Box 3. Guidance for practitioners on running a teleclinic

\section{Preparing before clinic (allow 7 minutes per patient)}

$>$ Review the relevant letters and results on electronic patient record (EPR) to get a feel for what to expect at consultation

Prepare a clinical note on EPR for recording the consultation

\section{Starting the consultation}

> Telephone or videophone the patient (if using a home landline number, use 141 before dialling)

> If a patient does not answer their phone, leave a message asking them to call your trust SIM number or the administrative team. If there is no answering service, you will need to write to the patient and advise them to make contact if clinically required

$>$ Give your full name and job role to the person answering, then ask to speak to the patient

$>$ Check that you are speaking to the actual patient and that the patient is in a suitable location to speak confidentially

$>$ Tell the patient that you have read their notes and say what you have concluded from these

$>$ Ask the patient about their condition and treatment

\section{Concluding the consultation}

$>$ Ask 'Is there anything else you want to ask me or to discuss?'

$>$ Summarise your conclusions and agree plans with patient, including follow-up. Specify which investigations, treatments or referrals you are requesting and how these are going to be carried out, including sending request forms or FP10 prescriptions to the patient with their copy of the clinic letter

$>$ Dictate a letter to the GP, with the letter copied to the patient

increased. Teleconsulting in some specialities is well-developed, notably in general practice, diabetes ${ }^{2}$ and dermatology, ${ }^{3}$ where video consulting is used to review existing skin lesions and to plan management of new skin disease for patients who are situated in locations that are remote or difficult to access, or who lack the ability to attend FTF clinics such as those living in care facilities. Primary Care Commissioning in combination with the British Association for Dermatology (BAD) has published Quality Standards for Teledermatology, ${ }^{4}$ with guidance updated in late March 2020 for the COVID-19 pandemic. ${ }^{5}$ The General Medical Council (GMC) has recently published guidance for the COVID-19 pandemic entitled Remote Consultations. ${ }^{6}$

Electronic consultation and telemedicine have long been used in sparsely populated large countries including Canada ${ }^{7}$ and Australia, ${ }^{8}$ while teleconsulting has been employed by general practitioners and has been in longer existence. However, many lessons from general practice are not fully generalisable to hospital outpatient services, where consultations are often not patient-initiated and involve detailed review and planning of the management of complex conditions with many relevant comorbidities.

With our rheumatology outpatient clinics overloaded through gradual accumulation of patients and long waiting times over the years, we had been considering introduction of teleclinics in order to triage and prioritise our patients with the greatest need while not neglecting the other patients, for whom we need to continue to provide specialist advice and monitoring.

The virtual clinics are part of the Trust Outpatients Transformation Programme. This is a systematic review of the delivery of our outpatient service within the Integrated Care Partnership. Patients were involved in the coproduction of the virtual clinics for the year prior to the first virtual clinic. Soon after we started trialling the virtual clinics at the start of 2020, the COVID-19 pandemic and the need for change in our working processes arrived rapidly in mid-March. The switch from FTF outpatient consultations to teleconsulting began on 18 March 2020. This offered opportunities to test plans that we had been considering for some time.

The digital readiness of our outpatient service meant we were very quickly able to provide virtual clinics as FTF clinics were restricted. We accelerated the deployment of equipment and
Attend Anywhere software to ensure patients could still have their clinically indicated reviews. In our Trust there was overall growth from $5 \%$ of clinics held virtually at the start of February 2020 to $42 \%$ by the end April 2020.

\section{Benefits of teleclinics}

We found that offsite teleclinics can shorten consultation time, as there are often fewer distractions and consultation can be more focused. We feel that routine booking of everyone who asks for an earlier appointment into a teleclinic avoids overloading of FTF clinics, with subsequent FTF appointments (especially if urgent) becoming more focused as those attending have already been screened and had provisional plans put in place.

Old age and presence of comorbidities were not a reason for us not to offer teleclinics. We found that this group were shielding at home during the pandemic and were available through this medium. The age range in our study was 17-92 years and the mean number of comorbidities was greater than one. The availability of telephone or video systems was more important than age or comorbidities. For older or immobile patients, we propose that remote consulting is dramatically easier than attending clinic and this may correct inequality of access.

We confirmed that teleclinics are useful for triage and support of patients with chronic pain problems, helping us to decide if inflammatory arthritis patients who had an increase in pain were indeed having a joint flare and would benefit most from an FTF appointment or a different approach. It was clear that a key benefit of teleclinics is in triage and streaming of patients, allowing direction of patients to the part of the service most appropriate to their needs. Future research will show if this reduces FTF clinic attendances and provides capacity to see new patients.

Teleclinics support patients on patient-initiated follow up (where the patient determines the need to come back to clinic) by allowing intermediate discussion encounters, as well as offering opportunities for other patients to discuss concerns they may have if there is a long interval before their next booked FTF appointment. All patients from teleclinics are considered for patient-initiated follow up if appropriate. Teleclinics promote 
Table 1. Patient demographics and outcome of the COVID-19 teleclinics

Patient demographics and characteristics

Number of patients in teleclinics, $\mathrm{n} \quad 396$

Age in years (mean, range) $\quad 58.9$ (17-924)

Gender (female:male) 280:116

Follow up: new appointments $\quad 350: 46$

Telephone: Video consultations $\quad 309: 87$

Number of comorbidities (mean, SD) $1.3(1.2)$

Diagnoses (n, \%)

Rheumatoid arthritis

Seronegative inflammatory arthritis

$130(32.8)$

Psoriatic arthritis

$44(11.1)$

47 (11.9)

25 (6.3)

$20(5.1)$

$37(9.4)$

Connective erythematosu

(not systemic lupus erythematosus)

Vasculitis (including Behcet's disease)

Crystal arthritis (gout)

$14(3.5)$

$7(2.3)$

$6(1.0)$

Juvenile idiopathic arthritis

$38(9.8)$

Giant cell arteritis and polymyalgia rheumatica

Fibromyalgia

$6(1.5)$

$7(1.8)$

$2(0.5)$

Osteoporosis

$13(4.0)$

\section{Outcome of teleclinics (n, \%)}

Patient discharged

39 (9.8)

No change in management, further routine

appointment booked

Change in management (tests ordered,

prescription issued)

Request to GP to give injection or treatment

Adjustment to treatment

(eg DMARD dose change)

Patient advised to see their GP due to issues

not related to their rheumatic disease or

its treatment

Referring the patient to another specialty

Referring patient to the Injection Clinic

Booking the patient into a face-to-face

clinic soon

Asking patient to go to $A \& E$ immediately or

arranging their admission through the acute

medical unit

Answering the patient's question and if

necessary offering them a follow-up phone

call in a week or two

Patient-initiated follow-up plan

$160(40.4)$

39 (9.8)

6 (1.5)

$102(25.8)$

$1(0.3)$

$5(1.3)$

$1(0.3)$

$10(2.5)$

$1(0.3)$

Unable to contact patient at time of

$22(5.6)$

teleconsultation

consultant and nurse ownership as all queries can be directed to the appropriate practitioner who will be able to deal directly with the patient.

We do not pretend that teleclinics take less time overall, but clearly they take up fewer of the hospital's physical resources, and the reduction in practitioner travelling time to our many outlying clinics would compensate for any extra time spent doing teleclinics. Teleclinics undertaken at our main base increases the availability of practitioners onsite for other tasks between teleconsultations. This supported clinicians who needed to be on the main base site during the pandemic. Most important decisions can be made, investigations initiated and vital triage performed at teleconsultation provided guidelines are followed. The decisions that can be made are shown in the results in Table 1.

\section{Risks of teleconsulting}

We found that with teleclinics confidentiality issues are paramount. It is impossible to be sure that one is maintaining confidentiality if one cannot see or hear who else is in the room. Only video software that meets NHS security standards should be used. The patient should not be in a location where they can be overheard. The video consultation is not recorded, just as FTF appointments are not recorded.

There may also be safeguarding issues if the patient is not able to be frank in the presence of others about problems they are having at home. Teleclinics may miss important physical signs that could only be picked up by hands-on physical examination. Video consultation nevertheless offers the possibility of picking up visual cues and may allow limited clinical examination. The absence of careful clinical examination may matter a great deal to many patients and may result in loss of patient confidence.

We also feel that situations that require the establishment of a relationship of trust between patient and practitioner are often not suitable for teleconsulting. For example, we are not yet satisfied that it is safe to initiate a new DMARD or biologic treatment without up-to-date hands-on clinical information, which can only be available at FTF consultation. This applies to many other situations of uncertainty where trust is required, such as challenging explanations of a new illness. This is an important factor to consider in new patient consultations. It is also likely that a lack of practitioner confidence or experience could be amplified in a teleconsultation, leading to a lack of patient confidence in the process.

There was limited time in the first few weeks of the pandemic to arrange translators or the presence of another household member to assist with language, hearing, vision and access to technology. There could have been possible selection bias as a result of this. In the latter half of the study and beyond, we have been able to arrange translators or another relative staying in the same home to assist in these areas and thus mitigate against the reduced access. Patients were asked if they had any disability that might prevent or hinder participation in virtual clinics and reasonable adjustments were made. While video consulting may well be beyond quite a lot of people, this applies less to phone consulting. Hearing problems were solved by having a partner or friend present with the patient's consent. As both telephone and video clinics are accessible through mobile phones or laptops, patients were given prior notice to find an area or room away from crowds. The mobile phones and laptops allowed patients increased mobility to participate in virtual clinics away from crowded living. We are aware that as the COVID-19 pandemic and lockdown eases, there may be less risk to attending for FTF appointment. We believe the case selection would not change significantly as the inclusion criteria for teleclinics (Box 2) are based on clinical indication instead of risk stratification against infection from COVID-19. We used the COVID-19 time to develop a protocol that we think will be suitable for use in the long term after the pandemic (Box 3).

We are evaluating the risks of teleconsulting by recording any harm that may have come to patients attending virtual clinics and 
comparing the frequency against FTF clinics. We have developed a risk register to record any harm or near misses from virtual clinics.

\section{Further evaluation}

There is huge potential for teleclinics to be implemented widely post COVID-19. However, further research on the long-term benefits, sustainability and limitations of teleconsultation in internal medical specialities is needed. Areas for future research include which patients prefer teleconsultations and which prefer FTF, and in what circumstances.

Clinical outcomes using patient-reported outcomes and experience measures (PROMS and PREMS) should be measured in teleclinics using FTF as controls, although selection bias may interfere with the results. Adverse events from teleclinics will need recording and evaluation.

Does teleconsulting reduce clinic workload, for example by reducing the number of FTF consultations per patient in the next 18 months compared with FTF? Again, selection bias may be an issue and will need to be considered. Time spent on FTF clinics versus teleconsulting (including all administration except checking and responding to results) will need to be assessed.

\section{Conclusion}

If teleconsultation is approached as another form of FTF consultation - with pre-consultation preparation, use of a consultation template rather than an unstructured conversation, and documentation to the usual standard - the majority of important decisions can be made, important investigations instituted and vital triage performed using teleconsultation.

Conducting these clinics from home during the pandemic felt less pressurised than outpatient clinics because the practitioner was aware that patients were waiting in their own homes rather than in a hospital waiting room.

It remains to be seen whether patients are satisfied with teleconsulting in the long term. Our anecdotal impression is that older patients who find any travelling difficult prefer teleconsultations to FTF consultations. Patients prefer to avoid attending clinics at main hospitals with parking difficulties, although, depending on their problems at the time of the clinic, many might prefer smaller peripheral clinics to teleclinics. Patients in future will be given the option of FTF or remote consultation. ${ }^{9}$ We are working towards ensuring that we maintain the current level of virtual clinics to deal with the backlog of appointments to be scheduled. This will aid in the recovery phase of the COVID-19 pandemic.

Our experience in implementing teleclinics during the COVID-19 pandemic has been positive. This technology has allowed bridging between accustomed clinical ways of working and the new consulting systems that we require. We have gained invaluable insight, learning and feedback that will inform our future delivery of outpatient clinics. The increased flexibility for practitioners offered by teleclinics tailored to their overall workload, together with the feedback from patients, suggests that this will be a successful change. For patients there is increased convenience in arranging appointments around their work and daily routine. For the Trust, the logistical and congestion challenges can be eased with reduced numbers of FTF appointments. Teleconsulting allows us to achieve the NHS Long Term Plan aim ${ }^{10,11}$ of reducing FTF appointments by a third over the next 5 years while improving patient experience and satisfaction.

\section{References}

1 Greenhalgh T, Vijayaraghavan S, Wherton ] et al. Virtual online consultations: advantages and limitations (VOCAL) study. BMJ Open 2016:6:e009388.

2 NHS England. Newham diabetes Skype pilot success increases young people's clinic attendance. www.england.nhs.uk/2016/01/ newham-diabetes-pilot-scheme/.

3 Edwards N, Imison C. How can dermatology services meet current and future patient needs, while ensuring quality of care is not compromised and access is equitable across the UK? Kings Fund, 2014. www.bad.org.uk/shared/get-file.ashx?id=2347\&itemtype=document.

4 British Association of Dermatologists. Quality standards for teledermatology. BAD, 2013. https://www.bad.org.uk/shared/get-file.ashx?itemtype= document\&id $=794$.

5 British Association of Dermatologists. COVID-19: Clinical guidelines for the management of dermatology patients remotely. BAD, 2020. www.bad.org.uk/healthcare-professionals/covid-19/ remote-dermatology-guidance.

6 General Medical Council. Remote consultations. www.gmc-uk.org/ ethical-guidance/ethical-hub/remote-consultations.

7 Liddy C, Bello A, Cook ] et al. Supporting the spread and scale-up of electronic consultation across Canada: cross-sectional analysis. BMJ Open 2019;9:e028888.

8 Bradford NK, Caffery LJ, Smith AC. Telehealth services in rural and remote Australia: a systematic review of models of care and fac tors influencing success and sustainability. Rural Remote Health 2016;16:3808

9 British Society for Rheumatology. Covid-19 remote consultations: principles for remote consultation. www.rheumatology.org.uk/ news-policy/details//COVID-19-remote-consultations.

10 NHS England. NHS Long Term Plan. NHSE, 2019. Available from www.longtermplan.nhs.uk/publication/nhs-long-term-plan/.

11 O'Cathail M, Sivanandan MA, Diver C et al. The use of patientfacing teleconsultations in the national health service: scoping review. JMIR Med Inform 2020;8:e15380.

Address for correspondence: Dr Antoni T Chan, Royal Berkshire Hospital, Royal Berkshire NHS Foundation Trust, London Road, Reading, RG1 5AN, UK.

Email: antoni.chan@nhs.net 